\title{
Comparative Risk Assessment of Severe Uterine Bleeding Following Exposure to Direct Oral Anticoagulants: A Network Study Across Four Observational Databases in the USA
}

\author{
James Weaver ${ }^{1}$ (D) Azza Shoaibi ${ }^{1}$ (D) Huy Q. Truong ${ }^{2} \cdot$ Leila Larbi $^{2} \cdot$ Shujian Wu $^{3} \cdot$ Peter Wildgoose $^{1} \cdot$ Gowtham Rao $^{1}$. \\ Amy Freedman ${ }^{1} \cdot$ Lu Wang $^{1}$ (D) Zhong Yuan ${ }^{1}$ (D) Elliot Barnathan ${ }^{2}$
}

Accepted: 16 February 2021 / Published online: 2 March 2021

(c) The Author(s) 2021

\begin{abstract}
Background Antithrombotic therapies are associated with an increased bleeding risk. Abnormal uterine bleeding data have been reported in clinical trials of patients with venous thromboembolism (VTE), but data are limited for patients with atrial fibrillation (AF).

Objective Using real-world data from four US healthcare databases (October 2010 to December 2018), we compared the occurrence of severe uterine bleeding among women newly exposed to rivaroxaban, apixaban, dabigatran, and warfarin stratified by indication.

Methods To reduce potential confounding, patients in comparative cohorts were matched on propensity scores. Treatment effect estimates were generated using Cox proportional hazard models for each indication, in each database, and only for pairwise comparisons that met a priori study diagnostics. If estimates were homogeneous $\left(I^{2}<40 \%\right)$, a meta-analysis across databases was performed and pooled hazard ratios reported.

Results Data from 363,919 women newly exposed to a direct oral anticoagulant or warfarin with a prior diagnosis of AF $(60.8 \%)$ or VTE $(39.2 \%)$ were analyzed. Overall incidence of severe uterine bleeding was low in the populations exposed to direct oral anticoagulants, although relatively higher in the younger VTE population vs the AF population (unadjusted incidence rates: $2.8-33.7$ vs $1.9-10.0$ events/1000 person-years). In the propensity score-matched AF population, a suggestive, moderately increased risk of severe uterine bleeding was observed for rivaroxaban relative to warfarin [hazard ratios and $95 \%$ confidence intervals from $0.83(0.27-2.48)$ to 2.84 (1.32-6.23) across databases with significant heterogeneity], apixaban [pooled hazard ratio 1.45 (0.91-2.28)], and dabigatran [2.12 (1.01-4.43)], which were sensitive to the time-atrisk period. In the propensity score-matched VTE population, a consistent increased risk of severe uterine bleeding was observed for rivaroxaban relative to warfarin [2.03 (1.19-3.27)] and apixaban [2.25 (1.45-3.41)], which were insensitive to the time-at-risk period.

Conclusions For women who need antithrombotic therapy, personalized management strategies with careful evaluation of benefits and risks are required.
\end{abstract}

ClinicalTrials.gov Registration NCT04394234; registered in May 2020.

Zhong Yuan

zyuan6@its.jnj.com

1 Janssen Research \& Development, LLC,

1125 Trenton-Harbourton Road, Titusville, NJ 08560, USA

2 Janssen Research \& Development, LLC, Raritan, NJ, USA

3 Janssen Research \& Development, LLC, Horsham, PA, USA 


\section{Key Points}

Using real-world data, the incidence of severe uterine bleeding was low but varied by antithrombotic therapy and occurred more often in patients with venous thromboembolism vs those with atrial fibrillation.

Compared with warfarin, apixaban, and dabigatran, rivaroxaban was associated with a moderately increased risk of severe uterine bleeding in patients with atrial fibrillation that was sensitive to the time-at-risk period analyzed and a more consistently increased risk in patients with venous thromboembolism regardless of the time-at-risk period analyzed.

Careful evaluation of the benefits and risks of antithrombotic therapy in women requires consideration of factors including age, comorbidities, treatment duration, and indication for use.

\section{Introduction}

Direct oral anticoagulants (DOACs), including rivaroxaban, apixaban, and dabigatran, are approved for use in patients with non-valvular atrial fibrillation $(\mathrm{AF})$ to prevent stroke and systemic embolism, in patients with venous thromboembolism (VTE) for the treatment and prevention of recurrent events, and in patients undergoing total knee or hip replacement surgery as prophylaxis against VTE [1-3]. Direct oral anticoagulants do not require routine laboratory monitoring and have fewer drug-drug and drug-food interactions, making them reliable and convenient alternatives to vitamin $\mathrm{K}$ antagonists, such as warfarin. Because of their biologic mechanism of action, all anticoagulants are associated with an increased risk of bleeding. In the ROCKET-AF trial of patients with non-valvular AF, major bleeding rates were similar between rivaroxaban and warfarin, but an increased risk of major gastrointestinal bleeding was observed in rivaroxaban-treated patients compared with warfarin-treated patients [2.0 vs 1.2 events/100 patient-years (PY), respectively; hazard ratio (HR) 1.66 ; $95 \%$ confidence interval (CI) 1.34-2.05] [4, 5].

Women of reproductive age have a higher risk of VTE compared with men of the same age, and this difference is attributed, in part, to the use of hormonal contraceptives and pregnancy [6]. Abnormal uterine bleeding events have been reported in clinical trials of DOACs for the treatment or prevention of VTE, and post hoc analyses of these trials have provided inconsistent results on the risk of abnormal uterine or vaginal bleeding among DOACs [7-10]. Two of these trials evaluated uterine bleeding with DOACs using the International Federation of Gynecology and Obstetrics criteria [8, 10], which defined abnormal uterine bleeding as prolonged menstrual bleeding; intermenstrual bleeding; heavy menstrual bleeding; or menstrual blood loss causing anemia or requiring an unscheduled contact with a physician, a medical or surgical intervention, or adaptation of anticoagulant therapy [11]. In contrast, abnormal uterine bleeding events have not been commonly reported in other populations, such as those with AF. One possible reason for the scarcity of information on uterine bleeding in clinical trials is that clinically relevant bleeding based on the International Society on Thrombosis and Haemostasis definition does not include the uterus as a critical organ [12]. Thus, the post hoc analyses of clinical trials are limited by the highly selected populations included in the trials and by differences in analysis design. Evidence from routine clinical practice across larger, more diverse populations, including different anticoagulant indications is lacking.

Therefore, the present, voluntary, sponsor-initiated study aimed to evaluate the risk of severe uterine bleeding, defined as vaginal bleeding requiring same-day transfusion or surgical management within 60 days, in relation to rivaroxaban, apixaban, dabigatran, and warfarin therapies. We conducted a set of retrospective comparative cohort analyses to assess whether individual DOACs (rivaroxaban, apixaban, and dabigatran) were associated with a risk of severe uterine bleeding compared with warfarin and with each other among women with prior diagnoses of the aforementioned clinical indications (AF and VTE, including deep vein thrombosis and pulmonary embolism) separately.

\section{Methods}

\subsection{Study Design}

This retrospective, observational, comparative, new-user cohort study evaluated data from adult women who were newly exposed to DOACs or warfarin in a population of insured patients in the USA (Fig. 1). The study was preregistered at ClinicalTrials.gov (NCT04394234), and the complete specification of all analyses is available in the protocol at https://github.com/ohdsi-studies/DoacsWarfa $\mathrm{rinSub} /$ tree/master/Protocol.

The overall study period was from 19 October 2010 (coinciding with initial approval of dabigatran) to 31 December 2018. In any pairwise comparison, the study period was restricted to calendar time when both exposures (drugs) were observed in each data source. 


\section{Eligibility criteria:}

- First exposure date to cohort-defining drug between 19 October 2010 and 31 December 2018

- No prior exposure to other study drugs or edoxaban

- $\geq 183$ days of prior continuous observation

- No prior hysterectomy, vaginal bleed, or related management

$\cdot \geq 1$ diagnosis of indication (e.g., AF) on or before index date

- No diagnoses of other indications

Medical history lookback time

Baseline covariates for confounding

adjustment from observed sources:

- Demographics (age, index year, and month)

- Prior conditions, drugs, procedures, and measurements observed during the 183 days before and including index date

- $\mathrm{CCl}, \mathrm{CHA}_{2} \mathrm{DS}_{2}$-VASc

- Number of distinct conditions, drugs, procedures, and visits observed in the prior 183 days
Causal contrasts of interest:

- On-treatment effect

- Sensitivity on-treatment effect

- Intent-to-treat effect

Rivaroxaban
Treatment strategies:

- Rivaroxaban

- Apixaban

- Dabigatran

- Warfarin

Fig. 1 Comparative cohort study design. $\mathrm{AF}$ atrial fibrillation, $\mathrm{CCI}$ Charlson Comorbidity Index, $\mathrm{CHA}_{2} \mathrm{DS} \mathrm{S}_{2}-\mathrm{VASc}$ stroke risk index, $\mathrm{PH}$ proportional hazards

\subsection{Data Sources}

This distributed database network study was executed using patient-level data from four US de-identified, administrative claims databases. All four databases have been standardized into the Observational Medical Outcomes Partnership Common Data Model (https://ohdsi.github. io/CommonDataModel/background.html) [13]. The databases included: (1) IBM MarketScan ${ }^{\circledR}$ Commercial Database (CCAE), a medical and drug insurance claims database of active employees, early retirees, Consolidated Omnibus Budget Reconciliation Act continuers, and their dependents insured by employer-sponsored plans; (2) IBM MarketScan ${ }^{\circledR}$ Multi-state Medicaid (MDCD), an administrative claims database containing the pooled healthcare experience of Medicaid enrollees from multiple states; (3) IBM MarketScan ${ }^{\circledR}$ Medicare Supplemental Beneficiaries (MDCR), an administrative health claims database for Medicare-eligible active and retired employees and their Medicare-eligible dependents from employer-sponsored supplemental plans; and (4) Optum's Clinformatics ${ }^{\circledR}$ Data Mart Database (Optum), an administrative health claims database for members who are fully insured in commercial plans or in administrative services only and commercial Medicare. Analyses of de-identified publicly available data do not constitute human subjects research and, as such, do not require institutional review board review/approval.

All analyses were performed independently within each of these four databases and no patient-level data were pooled across the databases for any analysis. Instead, meta-analysis estimates were computed based on per-database aggregate statistics as described below.

\subsection{Study Population}

Cohorts of mutually exclusive, adult (aged $\geq 18$ years) female new users of rivaroxaban, apixaban, dabigatran, and warfarin with $\geq 183$ days of prior continuous database observation were identified. The date of new anticoagulant dispensing was the index date. Patients were excluded for edoxaban exposure; exposure to other drugs of interest (e.g., DOAC users were excluded for the warfarin exposure cohort); hysterectomy; vaginal bleed; and medical, surgical, or transfusion management for vaginal bleeding any time prior to or on the index date. From these base exposure populations, additional inclusion criteria were added to construct new user cohorts with the following prior indications with consideration of the number of days prior to and including the index date: (1) AF population: AF diagnosis any time prior and no VTE in the past 183 days and no total knee or hip replacement surgery in the past 35 days; and (2) VTE population: VTE in the past 183 days, no AF diagnosis any time prior, and no total knee or hip replacement surgery in the past 35 days. All inclusion criteria are based on information referenced to the index date, such that "any time prior" means all observed time before and including the index date. In total, 12 new user cohorts were constructed for the three indications across four treatments. New user 
cohort definition details are available in the protocol Section 7.3 including code sets in Annex A and an example in Annex B (https://github.com/ohdsi-studies/DoacsWarfa rinSub/tree/master/Protocol/Tables_Annex).

\subsection{Outcome Assessment}

Patients with severe uterine bleeding were identified by having vaginal bleeding with a blood transfusion on the same day or surgical management on or within the subsequent 60 days, based on diagnostic and procedure codes recorded. Outcome ascertainment details are available in the protocol Section 7.4 and codes for the outcome definitions are available in Annex A (https://github.com/ ohdsi-studies/DoacsWarfarinSub/tree/master/Protocol/ Tables_Annex). Cases were identified based on patients' records provided by the source data and no additional case adjudication was performed.

Time-at-risk is the interval relative to the index date during which the outcome can be ascertained. We defined time-at-risk three ways in the study. The primary on-treatment time-at-risk period was defined as the time from 1 day after the anticoagulant index date to the end of inferred persistent exposure, the last day of observation, or the end of the study period, whichever came first. For DOACs, the final exposure record allowed for no more than a 3-day gap between successive exposure intervals (inferred by days' supply) plus 5 days appended to the last exposure date. This definition was intended to reflect the short half-life (approximately $12 \mathrm{~h}$ ) of DOACs. For warfarin, the final exposure record allowed for no more than a 7-day gap between successive exposure intervals (inferred by days' supply) plus 5 days appended to the last exposure date. This definition was intended to reflect the longer half-life (20-60 h) of warfarin.

Two sensitivity analyses were conducted using variations of time-at-risk periods to provide an increased time to observe severe uterine bleeding events. In the first sensitivity analysis, the on-treatment time-at-risk period was similarly defined as in the primary analysis, but the final exposure record allowed for no more than a 30-day gap between successive exposure intervals (inferred by days' supply) with no days appended to the last exposure date for DOACs and warfarin. This definition was intended to reflect the dispensing behavior in routine clinical practice. The second sensitivity analysis was an intent-to-treat (ITT) analysis, defined as the time from 1 day after the anticoagulant index date until the last day of observation or the end of the study period, whichever came first, regardless of the subsequent drug dispensing records. All time-at-risk period definitions required that patients have at least 1 day of time-at-risk after the index date.

\subsection{Statistical Analysis}

The unadjusted (crude) incidence rates (IRs) of severe uterine bleeding within each exposure cohort were reported and calculated as the number of persons with the outcome during each time-at-risk period, divided by the total time-at-risk (IR/1000 PY). Incidence proportion, defined as the number of persons with an outcome during the time-at-risk period divided by the total number of persons at risk, was also calculated. The number of severe uterine bleeding events observed during each time-at-risk period, total time-at-risk in years, and the IR and incidence proportion in the exposure cohorts for DOACs and warfarin were reported for each indication population in each database. The crude IRs do not reflect any statistical adjustment and are not intended for comparative purposes.

For population-level effect estimation, all pairwise comparisons of DOACs (rivaroxaban, apixaban, dabigatran) and warfarin were assessed nested within each of the two indication groups (AF or VTE). Propensity scores (PSs) were used to reduce potential confounding due to an imbalance of observed patient characteristics at baseline and were calculated for each patient using the predicted probability from a regularized logistic regression model, fit with a Laplace prior (LASSO) and the regularization hyperparameter selected by optimizing the likelihood in a tenfold cross validation [14-16]. The primary PS strategy matched the target to comparator patients in a 1:1 ratio and a sensitivity PS strategy variably matched the target to comparator patients in a maximum of a 1:100 ratio. Both approaches used a greedy matching algorithm with a caliper of 0.2 of the standard deviation on the logit scale of the PS distribution. Covariates used as input to the PS model included all available baseline covariates on demographics; prior conditions (including possible confounders such as cardiovascular comorbidities, polyp of cervix, endometriosis, other cancers, and pregnancy); medication use (including hormonal therapy drugs); procedures; and the risk scores, including the Charlson Comorbidity Index and the stroke risk index $\left(\mathrm{CHA}_{2} \mathrm{DS}_{2}\right.$-VASc), during the 183 days prior to or on the index date. Propensity score matching was evaluated based on a covariate balance before and after PS matching and considered successful when all standardized differences in means were $<0.1$ [17]. Study diagnostics (PS distribution, covariate balance, and empirical null distribution) for the primary analysis of each pairwise comparison for each database in AF and VTE populations are reported in the Electronic Supplementary Material (ESM). Because of limited dabigatran exposures in VTE, no formal comparisons were performed.

Cox proportional hazards regression models were used to model the time to the first outcome occurrence for the target relative to the comparator cohort in pairwise comparisons 
while accounting for the baseline covariate imbalance using the two PS matching strategies (1:1, unconditional; and 1:100, conditional) $[18,19]$. Empirical calibration using 127 negative control outcomes (ESM) was performed to account for residual error unaddressed by PS matching [20, 21]. In addition to negative control outcomes, we also generated and included synthetic positive control outcomes. Positive control outcomes were developed on the basis of the negative controls, but where the effect size was artificially increased to a predefined effect size by injection of additional simulated outcomes [21]. For each indication, estimates of severe uterine bleeding risk were reported as the empirically calibrated hazard ratio (cHR) with associated $95 \%$ calibrated CIs and $p$ values. For each indicationtarget-comparator-analysis combination, heterogeneity of the HRs across the four databases was estimated, using $I^{2}$ as a metric [22]. When there was sufficient homogeneity across sources $\left(I^{2}<40 \%\right)$ [23], database-specific estimates were pooled through a random effect meta-analysis using the Hartung-Knapp-Sidik-Jonkman inverse-variance method [24]. Pooled results including $p$ values corrected for multiple testing using Hochberg's step-up procedure are provided. We conducted this study using open-source software developed by the OHDSI community, CohortMethod (https://ohdsi. github.io/CohortMethod/) and Cyclops (https://ohdsi.githu b.io/Cyclops/) [25].

\section{Results}

The full result set with associated study diagnostics is publicly available in an interactive web application at https:// data.ohdsi.org/DoacsWarfarinSub/. Detailed results can be accessed by clicking on individual rows listed for each database under the "Explore Results" tab. This section summarizes the key findings.

A total of 363,919 women newly exposed to a DOAC or warfarin with a prior diagnosis of AF (60.8\%) or VTE (39.2\%) were included in the study, with the largest proportion from Optum (43.0\%) and followed by MDCR (25.3\%), CCAE (24.6\%), and MDCD (7.2\%) [ESM]. Treatment duration varied across indications and databases. For patients in the MDCD database, treatment duration was generally shorter than that for patients in other databases and sample size was also limited. For patients in other databases and with $\mathrm{AF}$, the median treatment duration for the primary analysis was 1.9-2.6 months for DOACs and 2.1-2.9 months for warfarin. Corresponding treatment durations for patients with VTE were 1.4-2.4 months and 2.2-2.4 months, respectively. As expected, the average (and median) treatment durations based on the on-treatment and ITT sensitivity analyses were longer than that for the primary on-treatment approach.

\subsection{AF}

\subsubsection{Study Participants}

Among patients with AF, median age varied slightly by exposure cohort, with new users of warfarin and apixaban being older (aged 60-80 years) than new users of dabigatran and rivaroxaban (aged 60-78 years) [ESM]. Table 1 illustrates the baseline characteristics of the AF study population by treatment exposure group for the pairwise comparison of rivaroxaban vs warfarin in the Optum database; data for the other five treatment comparisons are included in the ESM. Most patients in the four treatment cohorts had underlying heart disease, hypertension, and hyperlipidemia, and diabetes mellitus and osteoarthritis were also common. Consistent with these conditions, common baseline medications included beta-blockers, renin-angiotensin inhibitors, lipidmodifying agents, and diuretics. The online application is searchable for the baseline prevalence of any covariate by navigating to the "Population Characteristics" tab and selecting "Raw" view and searching for a covariate of interest.

\subsubsection{Incidence Rates of Severe Uterine Bleeding}

In the AF population, the unadjusted IRs during the primary on-treatment time-at-risk period ranged from 1.9 events/1000 PYs among warfarin new users in Optum to 10.0 events/1000 PYs among rivaroxaban new users in CCAE (Table 2). During sensitivity time-at-risk periods, IRs ranged from 1.6 to 12.1 events/1000 PYs when on-treatment was defined with 30-day persistence gaps and from 1.8 to 10.0 events/1000 PYs over the ITT follow-up. Across all AF cohorts, the unadjusted IRs were generally higher in the CCAE database and among rivaroxaban new users.

\subsubsection{Comparative Results}

For the AF population after PS matching, patient counts, event counts, calibrated HRs, and associated 95\% CIs for database-specific and meta-analytic estimates are reported for each pairwise comparison for the primary and sensitivity analyses in Fig. 2. Compared to warfarin, rivaroxaban was associated with cHRs (95\% CI) from 0.83 (0.27-2.48) to $2.84(1.32-6.23)$ in the primary analysis; a pooled estimate was not reported for this comparison because of observed heterogeneity across the databases. During longer time-at-risk periods, pooled cHRs $(95 \% \mathrm{CI})$ were 1.74 (1.20-2.50) and 1.55 (1.16-2.07) during the sensitivity ontreatment and ITT periods, respectively (Fig. 2a). Among new users of apixaban compared to warfarin, a suggestive increased risk of severe uterine bleeding was observed with a cHR (95\% CI) of $1.69(0.91-3.09)$ during the primary analysis. However, in the sensitivity 30-day on-treatment 
Table 1 Selected baseline characteristics. For the full set of baseline characteristics, visit the online application: https://data.ohdsi.org/Doacs WarfarinSub/. The online application is searchable for the baseline prevalence of any covariate by navigating to the "Population Characteristics" tab and selecting the "Raw" view and searching for a covariate of interest before and after 1:1 propensity score matching for the comparison of rivaroxaban vs warfarin in the atrial fibrillation population from the Optum database

\begin{tabular}{|c|c|c|c|c|c|c|}
\hline \multirow[t]{3}{*}{ Characteristic } & \multicolumn{3}{|c|}{ Before matching } & \multicolumn{3}{|c|}{ After matching } \\
\hline & \multirow{2}{*}{$\begin{array}{l}\text { Rivaroxaban }^{\mathrm{a}} \\
(n=21,858) \\
\%\end{array}$} & \multicolumn{2}{|l|}{$\begin{array}{l}\text { Warfarin } \\
(n=35,005)\end{array}$} & \multirow{2}{*}{$\begin{array}{l}\text { Rivaroxaban } \\
(n=13,891) \\
\%\end{array}$} & \multicolumn{2}{|l|}{$\begin{array}{l}\text { Warfarin } \\
(n=13,891)\end{array}$} \\
\hline & & $\%$ & Std. diff & & $\%$ & Std. diff \\
\hline \multicolumn{7}{|l|}{ Age group, years } \\
\hline $25-29$ & 0.0 & 0.0 & 0.01 & 0.0 & 0.0 & 0.01 \\
\hline $30-34$ & 0.1 & 0.1 & 0.01 & 0.1 & 0.0 & 0.02 \\
\hline $35-39$ & 0.2 & 0.1 & 0.01 & 0.2 & 0.1 & 0.02 \\
\hline $40-44$ & 0.4 & 0.2 & 0.02 & 0.3 & 0.2 & 0.03 \\
\hline $45-49$ & 0.8 & 0.4 & 0.04 & 0.5 & 0.4 & 0.02 \\
\hline $50-54$ & 1.9 & 1.1 & 0.06 & 1.4 & 1.3 & 0.00 \\
\hline $55-59$ & 3.9 & 2.5 & 0.08 & 2.6 & 2.5 & 0.01 \\
\hline $60-64$ & 6.6 & 4.7 & 0.08 & 5.1 & 4.9 & 0.01 \\
\hline $65-69$ & 14.8 & 11.7 & 0.09 & 12.8 & 12.9 & 0.00 \\
\hline $70-74$ & 18.8 & 17.1 & 0.04 & 18.5 & 18.5 & 0.00 \\
\hline $75-79$ & 18.7 & 19.5 & -0.02 & 19.5 & 19.4 & 0.00 \\
\hline $80-84$ & 18.2 & 27.6 & -0.22 & 21.3 & 22.0 & -0.02 \\
\hline $85-89$ & 15.7 & 14.9 & 0.02 & 17.6 & 17.7 & 0.00 \\
\hline \multicolumn{7}{|l|}{ Medical history: general } \\
\hline Acute respiratory disease & 24.5 & 27.0 & -0.06 & 24.8 & 25.1 & -0.01 \\
\hline Attention-deficit/hyperactivity disorder & 0.2 & 0.1 & 0.01 & 0.1 & 0.2 & -0.01 \\
\hline Chronic liver disease & 1.5 & 1.9 & -0.03 & 1.7 & 1.7 & 0.00 \\
\hline Chronic obstructive lung disease & 18.7 & 21.7 & -0.07 & 20.1 & 20.0 & 0.00 \\
\hline Crohn's disease & 0.3 & 0.4 & -0.01 & 0.3 & 0.5 & -0.02 \\
\hline Dementia & 6.1 & 6.9 & -0.03 & 6.9 & 6.7 & 0.01 \\
\hline Depressive disorder & 16.2 & 16.2 & 0.00 & 16.3 & 16.3 & 0.00 \\
\hline Diabetes mellitus & 30.4 & 35.1 & -0.10 & 31.8 & 31.8 & 0.00 \\
\hline Gastroesophageal reflux disease & 22.0 & 20.5 & 0.04 & 21.3 & 22.0 & -0.02 \\
\hline Gastrointestinal hemorrhage & 2.5 & 3.8 & -0.07 & 2.9 & 2.8 & 0.00 \\
\hline Human immunodeficiency virus infection & 0.1 & 0.0 & 0.01 & 0.1 & 0.0 & 0.01 \\
\hline Hyperlipidemia & 64.2 & 64.1 & 0.00 & 64.2 & 64.3 & 0.00 \\
\hline Hypertensive disorder & 82.0 & 83.6 & -0.04 & 83.2 & 83.1 & 0.00 \\
\hline Lesion of liver & 1.0 & 1.2 & -0.02 & 1.1 & 1.0 & 0.01 \\
\hline Obesity & 18.5 & 15.2 & 0.09 & 16.7 & 16.8 & 0.00 \\
\hline Osteoarthritis & 32.7 & 30.9 & 0.04 & 32.3 & 32.4 & 0.00 \\
\hline Pneumonia & 10.3 & 13.4 & -0.09 & 11.5 & 11.3 & 0.01 \\
\hline Psoriasis & 1.2 & 0.8 & 0.04 & 1.0 & 0.9 & 0.01 \\
\hline Renal impairment & 20.9 & 29.2 & -0.19 & 23.9 & 23.4 & 0.01 \\
\hline Rheumatoid arthritis & 2.9 & 3.2 & -0.01 & 3.1 & 3.2 & 0.00 \\
\hline Schizophrenia & 0.2 & 0.2 & 0.01 & 0.2 & 0.2 & 0.00 \\
\hline Ulcerative colitis & 0.4 & 0.4 & 0.00 & 0.5 & 0.4 & 0.01 \\
\hline Urinary tract infectious disease & 17.4 & 21.4 & -0.10 & 18.8 & 18.7 & 0.00 \\
\hline Viral hepatitis C & 0.4 & 0.4 & 0.00 & 0.4 & 0.4 & 0.01 \\
\hline Visual system disorder & 37.6 & 37.8 & 0.00 & 38.4 & 37.8 & 0.01 \\
\hline \multicolumn{7}{|l|}{ Medical history: cardiovascular disease } \\
\hline Atrial fibrillation & 94.5 & 94.7 & -0.01 & 94.1 & 94.2 & 0.00 \\
\hline Cerebrovascular disease & 15.3 & 22.2 & -0.18 & 17.4 & 17.2 & 0.01 \\
\hline Coronary arteriosclerosis & 24.6 & 32.0 & -0.16 & 27.1 & 26.8 & 0.01 \\
\hline
\end{tabular}


Table 1 (continued)

\begin{tabular}{|c|c|c|c|c|c|c|}
\hline \multirow[t]{3}{*}{ Characteristic } & \multicolumn{3}{|c|}{ Before matching } & \multicolumn{3}{|c|}{ After matching } \\
\hline & \multirow{2}{*}{$\begin{array}{l}\text { Rivaroxaban }^{\mathrm{a}} \\
(n=21,858) \\
\%\end{array}$} & \multicolumn{2}{|l|}{$\begin{array}{l}\text { Warfarin }^{\mathrm{b}} \\
(n=35,005)\end{array}$} & \multirow{2}{*}{$\begin{array}{l}\text { Rivaroxaban } \\
(n=13,891) \\
\%\end{array}$} & \multirow{2}{*}{$\begin{array}{l}\text { Warfarin } \\
(n=13,891) \\
\%\end{array}$} & \multirow[b]{2}{*}{ Std. diff } \\
\hline & & $\%$ & Std. diff & & & \\
\hline Heart disease & 98.4 & 98.7 & -0.02 & 98.3 & 98.5 & -0.02 \\
\hline Heart failure & 27.6 & 37.8 & -0.22 & 31.3 & 31.0 & 0.01 \\
\hline Ischemic heart disease & 16.5 & 21.4 & -0.12 & 18.1 & 18.4 & -0.01 \\
\hline Peripheral vascular disease & 8.3 & 10.1 & -0.06 & 9.2 & 8.7 & 0.02 \\
\hline Venous thrombosis & 0.7 & 0.9 & -0.02 & 0.8 & 0.9 & -0.01 \\
\hline \multicolumn{7}{|l|}{ Medical history: neoplasms } \\
\hline Hematologic neoplasm & 1.6 & 1.9 & -0.02 & 1.7 & 1.8 & 0.00 \\
\hline Malignant lymphoma & 0.9 & 0.8 & 0.00 & 0.8 & 0.9 & -0.01 \\
\hline Malignant neoplasm of anorectum & 0.2 & 0.2 & -0.01 & 0.2 & 0.2 & 0.01 \\
\hline Malignant neoplastic disease & 11.7 & 12.0 & -0.01 & 11.9 & 11.8 & 0.00 \\
\hline Malignant tumor of breast & 4.0 & 3.8 & 0.01 & 3.9 & 3.9 & 0.00 \\
\hline Malignant tumor of colon & 0.6 & 0.8 & -0.02 & 0.7 & 0.7 & 0.00 \\
\hline Malignant tumor of lung & 0.8 & 0.9 & 0.00 & 1.0 & 0.9 & 0.01 \\
\hline Malignant tumor of urinary bladder & 0.3 & 0.3 & -0.01 & 0.2 & 0.3 & -0.01 \\
\hline Primary malignant neoplasm of prostate & 0.0 & 0.0 & 0.00 & 0.0 & 0.0 & -0.01 \\
\hline \multicolumn{7}{|l|}{ Medication use } \\
\hline Agents acting on the renin-angiotensin system & 55.5 & 56.1 & -0.01 & 57.2 & 57.0 & 0.00 \\
\hline Antibacterials for systemic use & 48.9 & 47.9 & 0.02 & 48.7 & 49.1 & -0.01 \\
\hline Antidepressants & 28.2 & 27.7 & 0.01 & 28.3 & 28.3 & 0.00 \\
\hline Antiepileptics & 16.2 & 15.7 & 0.01 & 16.3 & 16.5 & -0.01 \\
\hline Anti-inflammatory and antirheumatic products & 18.8 & 15.4 & 0.09 & 17.7 & 17.4 & 0.01 \\
\hline Antineoplastic agents & 3.9 & 3.8 & 0.01 & 4.0 & 4.0 & 0.00 \\
\hline Antipsoriatics & 0.6 & 1.3 & -0.06 & 0.7 & 0.8 & -0.02 \\
\hline Antithrombotic agents & 17.2 & 23.1 & -0.15 & 19.0 & 18.6 & 0.01 \\
\hline Beta-blocking agents & 70.6 & 70.9 & 0.00 & 71.3 & 71.4 & 0.00 \\
\hline Calcium channel blockers & 44.6 & 44.6 & 0.00 & 45.1 & 45.2 & 0.00 \\
\hline Diuretics & 51.6 & 57.4 & -0.12 & 54.2 & 54.2 & 0.00 \\
\hline Drugs for acid-related disorders & 32.8 & 33.4 & -0.01 & 33.2 & 33.7 & -0.01 \\
\hline Drugs for obstructive airway diseases & 28.7 & 25.9 & 0.06 & 27.4 & 27.6 & 0.00 \\
\hline Drugs used in diabetes mellitus & 20.8 & 23.7 & -0.07 & 21.5 & 21.7 & 0.00 \\
\hline Immunosuppressants & 3.6 & 3.0 & 0.04 & 3.4 & 3.4 & 0.00 \\
\hline Lipid-modifying agents & 53.9 & 56.4 & -0.05 & 55.7 & 55.4 & 0.01 \\
\hline Opioids & 22.1 & 24.1 & -0.05 & 22.5 & 22.8 & -0.01 \\
\hline Psycholeptics & 26.1 & 23.8 & 0.05 & 25.2 & 25.5 & -0.01 \\
\hline $\begin{array}{l}\text { Psychostimulants, agents used for attention-deficit/ } \\
\text { hyperactivity disorder and nootropics }\end{array}$ & 1.0 & 0.7 & 0.03 & 0.9 & 0.9 & 0.00 \\
\hline Risk scores & Mean & Mean & Std. diff & Mean & Mean & Std. diff \\
\hline Charlson Comorbidity Index & 3.79 & 4.43 & -0.20 & 4.08 & 4.06 & 0.00 \\
\hline $\mathrm{CHA}_{2} \mathrm{DS}_{2}$-VASc & 4.69 & 5.20 & -0.31 & 4.94 & 4.94 & 0.00 \\
\hline
\end{tabular}

$\mathrm{CHA}_{2} \mathrm{DS}_{2}-V A S c$ stroke risk index, Std. diff standardized difference

${ }^{a}$ In the rivaroxaban group, 5 patients were excluded from the study for not having $\geq 1$ day time-at-risk

${ }^{b}$ In the warfarin group, 14 patients were excluded from the study for not having $\geq 1$ day time-at-risk and 6200 patients were excluded when restricting patients to the calendar time when both exposures were observed in the database 


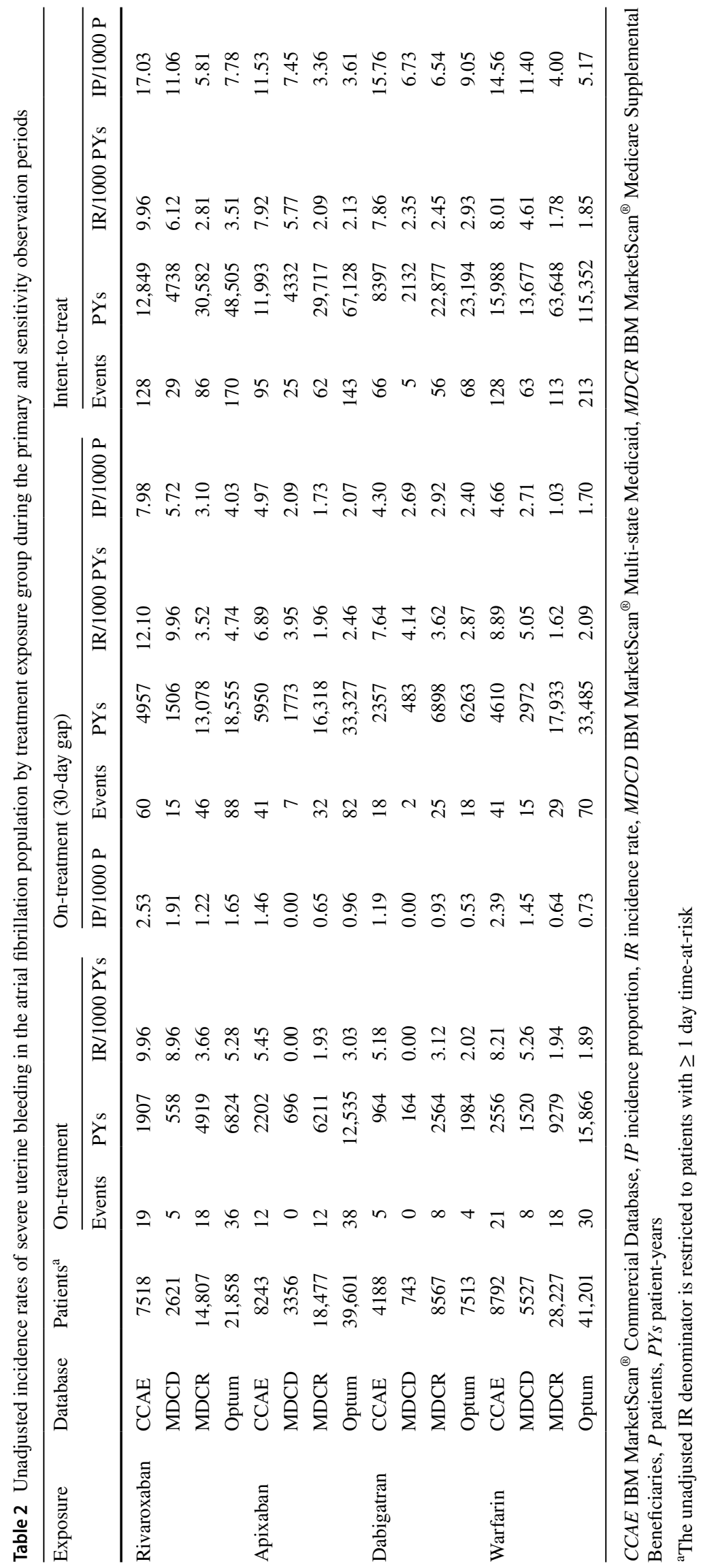


Fig. 2 Calibrated hazard ratios and calibrated $95 \%$ confidence intervals (CIs) for each pairwise comparison during primary and sensitivity observation periods in the atrial fibrillation (AF) population after $1: 1$ propensity score matching: (a) rivaroxaban vs warfarin; (b) apixaban vs warfarin; (c) dabigatran vs warfarin; (d) rivaroxaban vs apixaban; (e) rivaroxaban vs dabigatran; and (f) apixaban vs dabigatran. Estimates are reported for pairwise comparisons in databases where study diagnostic passed. Summary meta-analytic estimates are reported where $I^{2}<40 \%$.

CCAE IBM MarketScan ${ }^{\circledR}$

Commercial Database, $c H R$ calibrated hazard ratio, ITT intent-to-treat, $M D C D$ IBM MarketScan ${ }^{\circledR}$ Multi-state Medicaid, MDCR IBM

MarketScan ${ }^{\circledR}$ Medicare Supplemental Beneficiaries

\begin{tabular}{|c|c|c|c|c|c|c|c|}
\hline (a) & & & Number $\mathrm{c}$ & vents & & & \\
\hline Time-at-risk & Database & matched pairs & Rivaroxaban & Warfarin & $\mathrm{cHR}(95 \% \mathrm{Cl})$ & & \\
\hline On-treatment & CCAE & 2909 & 6 & 9 & $0.83(0.27-2.48)$ & $\longrightarrow$ & - \\
\hline & MDCR & 9362 & 12 & 5 & $2.51(0.83-7.87)$ & & \\
\hline & Optum & 13,891 & 22 & 11 & $2.84(1.32-6.23)$ & & $\longrightarrow$ \\
\hline On-treatment & CCAE & 2909 & 25 & 17 & $1.30(0.65-2.62)$ & - & $\rightarrow$ \\
\hline (30-day gap) & MDCR & 9362 & 28 & 9 & $2.31(1.04-5.24)$ & & \\
\hline & Optum & 13,891 & 54 & 28 & $1.87(1.17-2.98)$ & & $\longrightarrow$ \\
\hline & Summary & 26,162 & 107 & 54 & $1.74(1.20-2.50)$ & & $\leadsto$ \\
\hline ITT & CCAE & 2909 & 53 & 45 & $1.21(0.77-1.92)$ & & $\rightarrow$ \\
\hline & MDCR & 9362 & 54 & 29 & $1.86(1.16-3.03)$ & & $\longrightarrow$ \\
\hline & Optum & 13,891 & 119 & 76 & $1.64(1.22-2.22)$ & & $\rightarrow$ \\
\hline & Summary & 26,162 & 226 & 150 & $1.55(1.16-2.07)$ & & $\leadsto$ \\
\hline & & & & & & $\begin{array}{ccc} & 1 & 1 \\
0.1750 .25 & 0.5\end{array}$ & $\begin{array}{ll}1 & 1 \\
4 & 6\end{array}$ \\
\hline
\end{tabular}

(b)

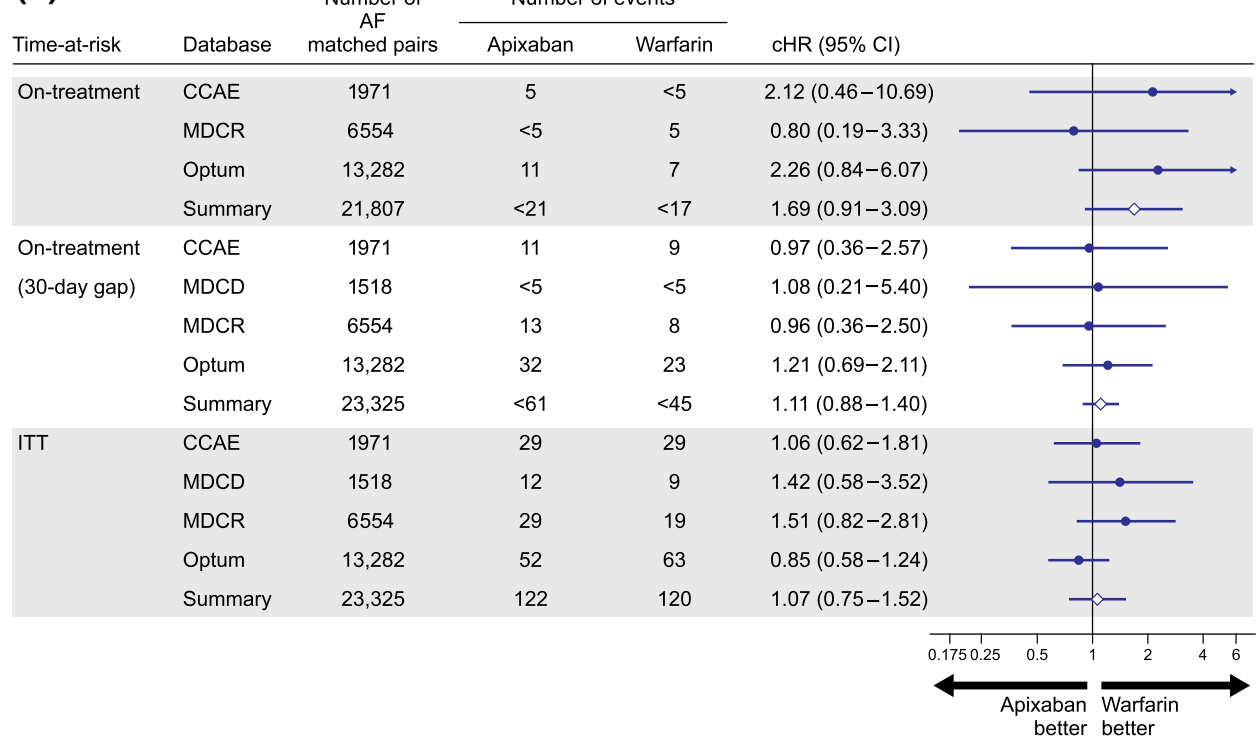

(c)

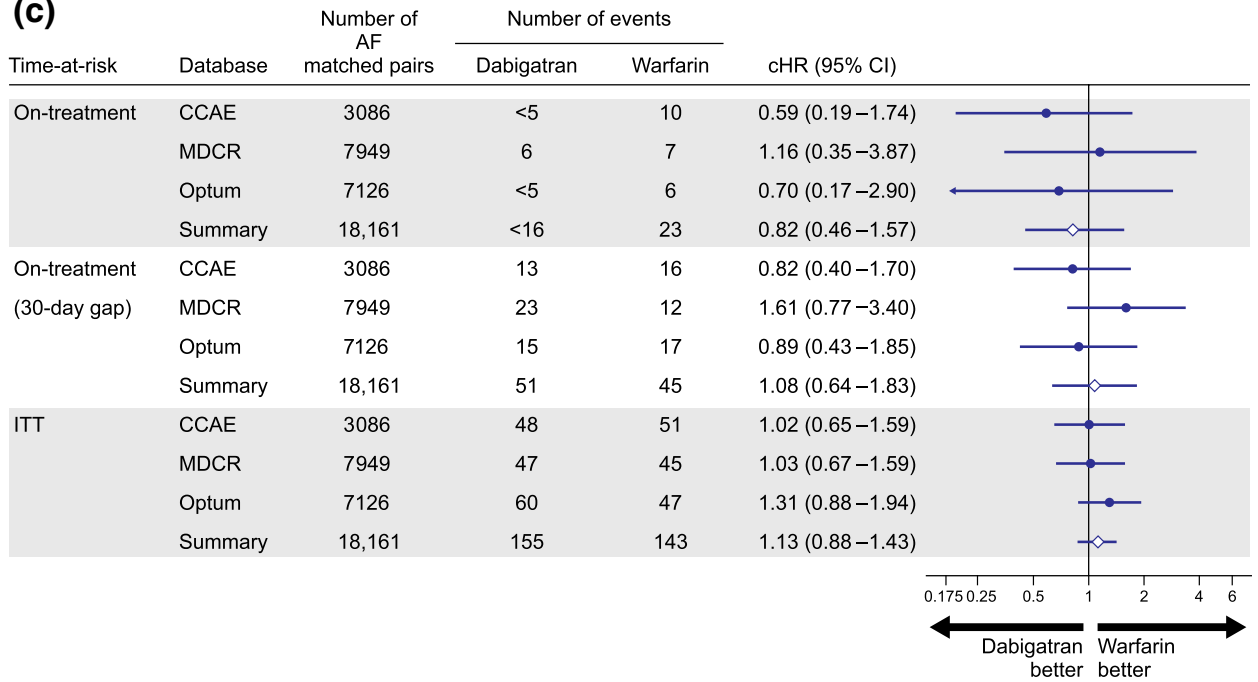


Fig. 2 (continued)

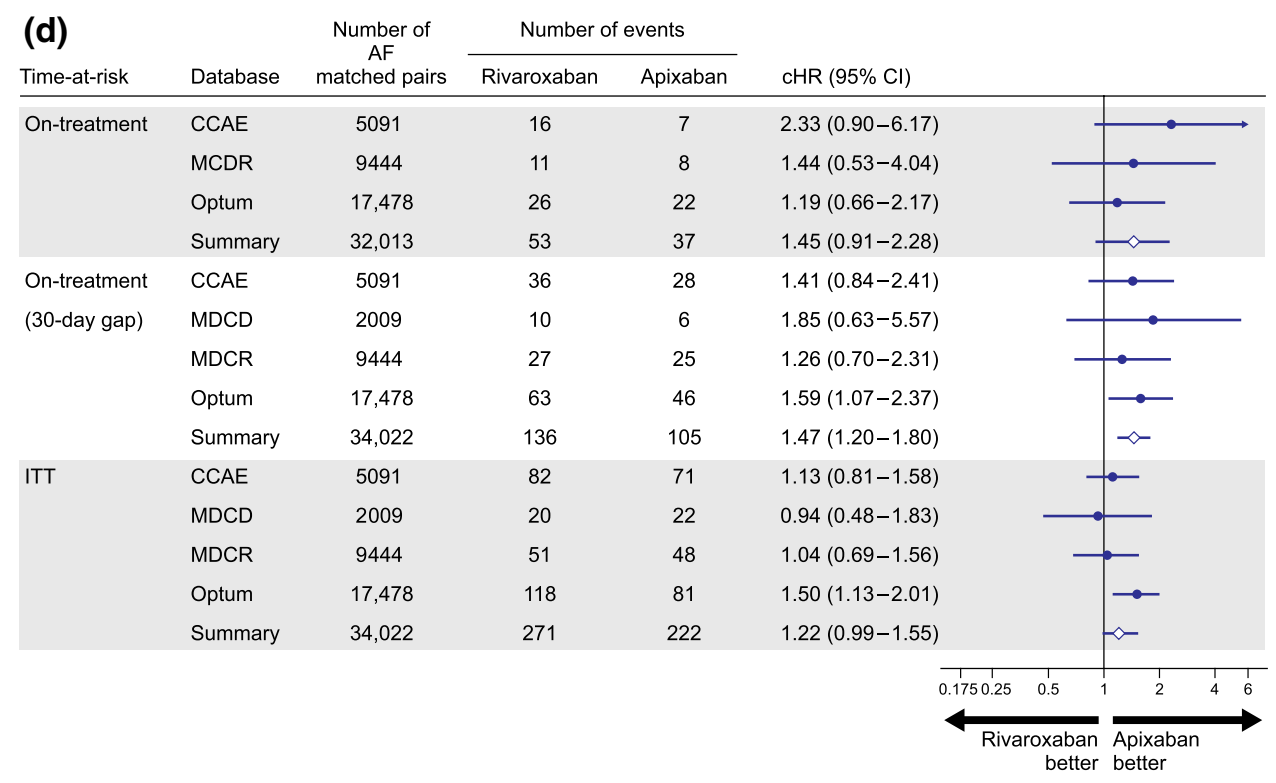

\begin{tabular}{|c|c|c|c|c|c|c|c|}
\hline \multirow{2}{*}{$\begin{array}{l}\text { (e) } \\
\text { Time-at-risk }\end{array}$} & \multirow[b]{2}{*}{ Database } & \multirow{2}{*}{$\begin{array}{c}\text { Number of } \\
A F \\
\text { matched pairs }\end{array}$} & \multicolumn{2}{|c|}{ Number of events } & \multirow[b]{2}{*}{ cHR $(95 \% \mathrm{Cl})$} & & \\
\hline & & & Rivaroxaban & $\overline{\text { Dabigatran }}$ & & & \\
\hline \multirow[t]{3}{*}{ On-treatment } & MDCR & 4028 & 7 & $<5$ & $1.67(0.46-6.21)$ & & . \\
\hline & Optum & 4729 & 10 & $<5$ & $3.00(0.77-11.87)$ & & \\
\hline & Summary & 8757 & 17 & $<10$ & $2.12(1.01-4.43)$ & & \\
\hline On-treatment & CCAE & 1972 & 20 & 6 & $2.78(1.10-7.31)$ & & $\longrightarrow$ \\
\hline \multirow[t]{3}{*}{ (30-day gap) } & MDCR & 4028 & 17 & 12 & $1.22(0.58-2.60)$ & & . \\
\hline & Optum & 4729 & 23 & 12 & $1.65(0.80-3.43)$ & & \\
\hline & Summary & 10,729 & 60 & 30 & $1.62(0.94-2.72)$ & & $\sim$ \\
\hline \multirow[t]{4}{*}{ ITT } & CCAE & 1972 & 38 & 24 & $1.58(0.94-2.68)$ & & $\longrightarrow$ \\
\hline & MDCR & 4028 & 30 & 24 & $1.32(0.77-2.27)$ & & - \\
\hline & Optum & 4729 & 57 & 43 & $1.33(0.89-1.99)$ & & 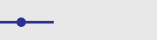 \\
\hline & Summary & 10,729 & 125 & 91 & $1.40(1.07-1.79)$ & & 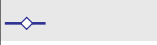 \\
\hline & & & & & & $\begin{array}{ll} & 1 \\
0.1750 .25 & 0.5\end{array}$ & $\frac{1}{4}$ \\
\hline
\end{tabular}

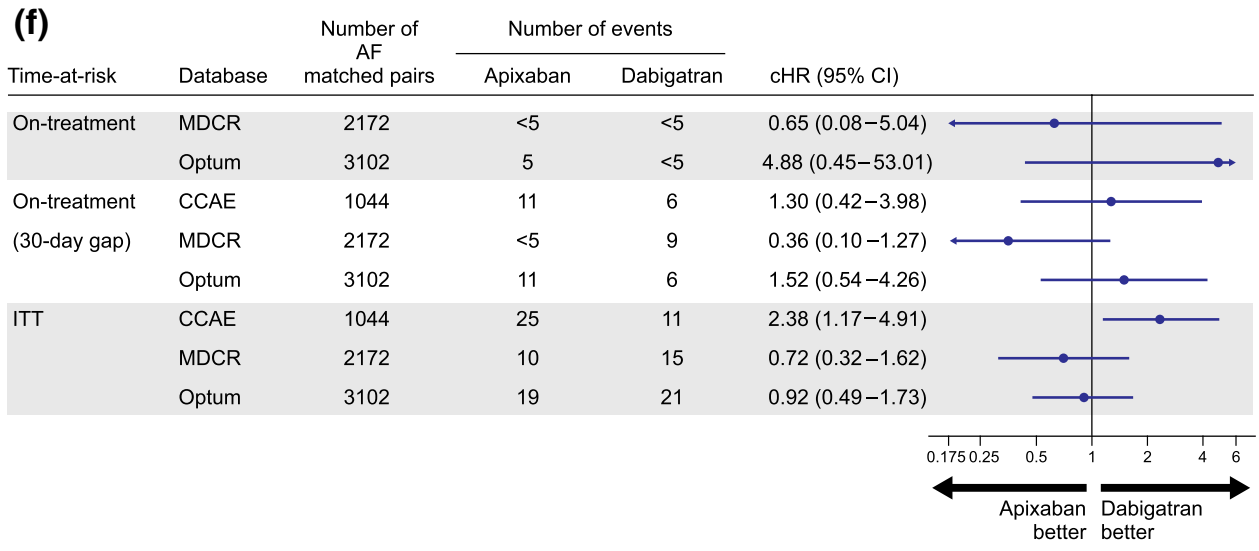

[1.11 (0.88-1.40)] and ITT [1.07 (0.75-1.52)] analyses, the estimates were attenuated towards the null (Fig. 2b). For new users of dabigatran compared to warfarin, no difference in the risk of severe uterine bleeding was observed, and uncertainty decreased with increased time-at-risk
(Fig. 2c). A non-significant increased risk of severe uterine bleeding among rivaroxaban new users compared to apixaban new users was observed in the primary analysis with a pooled cHR $(95 \% \mathrm{CI})$ of $1.45(0.91-2.28)$. Increased time-at-risk decreased the variance of the estimate to reach 
statistical significance, with a pooled cHR $(95 \% \mathrm{CI})$ of 1.47 (1.20-1.80) during the sensitivity on-treatment period and 1.22 (0.99-1.55) during the ITT period (Fig. 2d). New use of rivaroxaban compared with dabigatran was associated with a cHR (95\% CI) of $2.12(1.01-4.43)$ in the primary analysis, 1.62 (0.94-2.72) during the sensitivity on-treatment period, and $1.40(1.07-1.79)$ during the ITT period (Fig. 2e). The risk of severe uterine bleeding in apixaban compared to dabigatran new users was highly uncertain during primary and sensitivity on-treatment periods and suggestive of no risk difference during the ITT period (Fig. 2f).

\subsection{VTE}

\subsubsection{Study Population}

Among patients with VTE, median age varied slightly by cohort, with apixaban (51-78 years) and dabigatran new users the oldest (50-78 years) compared with new users of warfarin (50-77 years) and rivaroxaban (48-76 years) [ESM]. Table 3 illustrates the baseline characteristics of the VTE study population by treatment exposure group for the pairwise comparison of rivaroxaban vs warfarin in the Optum database; data for the other five treatment comparisons are included in the ESM. Most patients had hypertension and approximately half had underlying heart disease and hyperlipidemia. Other common baseline comorbidities included osteoarthritis, acute respiratory disease, gastrointestinal reflux disease, diabetes mellitus, and renal impairment. The most prescribed baseline medications included systemic antibiotics, renin-angiotensin inhibitors, lipidmodifying agents, diuretics, opioids, and drugs for acidrelated disorders. As noted previously, other covariates can be viewed in the online application.

\subsubsection{Incidence Rates of Severe Uterine Bleeding}

In the VTE population during the primary on-treatment time-at-risk period, unadjusted IRs ranged from 2.8 events/1000 PYs among apixaban new users in MDCR to 33.7 events/1000 PYs among rivaroxaban new users in CCAE (Table 4). During sensitivity time-at-risk periods, IRs ranged from 2.3 to 25.6 events/1000 PYs when on-treatment was defined with 30-day persistence gaps and from 2.0 to 14.1 events/1000 PYs over the ITT follow-up. Small sample sizes and zero-event counts precluded generating meaningful IRs for dabigatran-exposed women with VTE.

\subsubsection{Comparative Results}

For the VTE population after PS matching, patient counts, event counts, calibrated HRs, and associated 95\% CIs for database-specific and meta-analytic estimates are reported for each pairwise comparison for the primary and sensitivity analyses in Fig. 3. In the primary analysis, a twofold increased risk of severe uterine bleeding was observed among rivaroxaban new users compared to warfarin new users, with a pooled cHR (95\% CI) of 2.03 (1.19-3.27). The risk was consistently elevated across time-at-risk definitions with slight attenuation as time-at-risk increased. The pooled cHR (95\% CI) was 1.84 (1.38-2.37) during the sensitivity on-treatment period and $1.32(1.06-1.65)$ during the ITT period (Fig. 3a). New use of apixaban compared with warfarin was associated with a cHR (95\% CI) of $1.13(0.55-2.28)$ in the primary analysis, $1.14(0.73-1.76)$ during the sensitivity on-treatment period and $1.00(0.81-1.22)$ during the ITT period (Fig. 3b). Compared to apixaban, rivaroxaban was associated with cHR (95\% CI) of 2.25 (1.45-3.41) for severe uterine bleeding, which was consistently observed during the sensitivity 30-day on-treatment period, with a cHR (95\% CI) of 2.32 (1.41-3.59). An increased risk was also observed during the ITT period, albeit attenuated to 1.63 (1.11-2.45) (Fig. 3c).

\subsection{Other Analyses}

Results from the sensitivity PS matching strategy (1:100) were qualitatively similar to the primary PS approach in all comparisons (see ESM for AF and VTE populations).

\section{Discussion}

Using real-world data from routine clinical practice, we evaluated and compared the occurrence of severe uterine bleeding in relation to the use of rivaroxaban, apixaban, dabigatran, and warfarin for patients with AF and VTE. The overall incidence of severe uterine bleeding was low in the populations exposed to DOACs, although relatively higher in the younger VTE population than in the AF population (unadjusted IRs: $2.9-34.2$ vs $1.9-10.1$ events/ $1000 \mathrm{PYs}$ ). Among patients with AF, the risk of severe uterine bleeding was statistically comparable among all treatment groups in the main analysis. However, a trend toward an increased risk was observed among new users of rivaroxaban as compared to all other drugs. These results were sensitive to the time-at-risk periods. The sensitivity analyses of the on-treatment period allowing a 30-day gap between successive exposure intervals and the ITT period resulted in a statistically significant increased risk of severe uterine bleeding among new users of rivaroxaban vs warfarin with a calibrated pooled HR of 1.74 and 1.55 , respectively. In the sensitivity on-treatment analysis, new users of rivaroxaban were also at a higher risk of severe uterine bleeding compared to apixaban, with a calibrated pooled HR of 1.47. In general, the statistically significant pooled HR was driven 
Table 3 Selected baseline characteristics. For the full set of baseline characteristics, visit the online application: https://data.ohdsi.org/Doacs WarfarinSub/. The online application is searchable for the baseline prevalence of any covariate by navigating to the "Population Characteristics" tab and selecting the "Raw" view and searching for a covariate of interest before and after 1:1 propensity score matching for the comparison of rivaroxaban vs warfarin in the venous thromboembolism population from the Optum database

\begin{tabular}{|c|c|c|c|c|c|c|}
\hline \multirow[t]{3}{*}{ Characteristic } & \multicolumn{3}{|c|}{ Before matching } & \multicolumn{3}{|c|}{ After matching } \\
\hline & \multirow{2}{*}{$\begin{array}{l}\text { Rivaroxaban } \\
(n=14,480)^{\mathrm{a}}\end{array}$} & \multicolumn{2}{|l|}{$\begin{array}{l}\text { Warfarin } \\
(n=25,972)^{\mathrm{b}}\end{array}$} & \multirow{2}{*}{$\begin{array}{l}\text { Rivaroxaban } \\
(n=6921) \\
\%\end{array}$} & \multirow{2}{*}{$\begin{array}{l}\text { Warfarin } \\
(n=6921) \\
\%\end{array}$} & \multirow[b]{2}{*}{ Std. diff } \\
\hline & & $\%$ & Std. diff & & & \\
\hline \multicolumn{7}{|l|}{ Age group, years } \\
\hline $20-24$ & 0.7 & 0.5 & 0.02 & 0.8 & 0.4 & 0.05 \\
\hline $25-29$ & 1.0 & 0.9 & 0.02 & 0.9 & 0.7 & 0.02 \\
\hline $30-34$ & 1.3 & 1.3 & 0.00 & 1.1 & 1.3 & -0.02 \\
\hline $35-39$ & 2.6 & 2.0 & 0.04 & 2.2 & 2.1 & 0.00 \\
\hline $40-44$ & 3.5 & 2.9 & 0.04 & 3.0 & 2.5 & 0.03 \\
\hline $45-49$ & 5.0 & 4.1 & 0.04 & 4.0 & 4.1 & -0.01 \\
\hline $50-54$ & 6.5 & 5.3 & 0.05 & 5.3 & 5.2 & 0.00 \\
\hline $55-59$ & 8.8 & 7.2 & 0.06 & 7.7 & 7.2 & 0.02 \\
\hline $60-64$ & 10.1 & 9.0 & 0.04 & 8.9 & 9.0 & 0.00 \\
\hline $65-69$ & 14.2 & 14.0 & 0.01 & 14.1 & 15.0 & -0.02 \\
\hline $70-74$ & 15.0 & 14.3 & 0.02 & 14.8 & 15.6 & -0.02 \\
\hline $75-79$ & 11.9 & 12.8 & -0.03 & 13.1 & 13.1 & 0.00 \\
\hline $80-84$ & 9.7 & 16.9 & -0.21 & 12.4 & 11.7 & 0.02 \\
\hline $85-89$ & 9.7 & 8.6 & 0.04 & 11.8 & 11.9 & 0.00 \\
\hline \multicolumn{7}{|l|}{ Medical history: general } \\
\hline Acute respiratory disease & 28.8 & 30.7 & -0.04 & 31.0 & 31.0 & 0.00 \\
\hline Attention-deficit/hyperactivity disorder & 0.8 & 0.5 & 0.03 & 0.6 & 0.7 & -0.01 \\
\hline Chronic liver disease & 2.1 & 3.7 & -0.10 & 2.7 & 3.4 & -0.04 \\
\hline Chronic obstructive lung disease & 17.4 & 20.5 & -0.08 & 20.2 & 20.3 & 0.00 \\
\hline Crohn's disease & 0.9 & 1.0 & -0.01 & 1.0 & 1.0 & 0.00 \\
\hline Dementia & 8.4 & 10.5 & -0.07 & 11.0 & 10.4 & 0.02 \\
\hline Depressive disorder & 24.1 & 24.0 & 0.00 & 26.2 & 26.2 & 0.00 \\
\hline Diabetes mellitus & 26.0 & 30.1 & -0.09 & 29.6 & 29.1 & 0.01 \\
\hline Gastroesophageal reflux disease & 27.3 & 26.4 & 0.02 & 28.8 & 29.1 & -0.01 \\
\hline Gastrointestinal hemorrhage & 4.4 & 6.2 & -0.08 & 5.5 & 5.8 & -0.02 \\
\hline Human immunodeficiency virus infection & 0.2 & 0.2 & -0.01 & 0.2 & 0.2 & 0.00 \\
\hline Hyperlipidemia & 49.0 & 51.4 & -0.05 & 52.7 & 52.0 & 0.01 \\
\hline Hypertensive disorder & 64.0 & 70.5 & -0.14 & 69.8 & 69.6 & 0.00 \\
\hline Lesion of liver & 4.2 & 3.9 & 0.02 & 4.3 & 4.3 & 0.00 \\
\hline Obesity & 23.4 & 20.7 & 0.07 & 23.8 & 24.8 & -0.02 \\
\hline Osteoarthritis & 40.1 & 38.1 & 0.04 & 41.3 & 41.1 & 0.00 \\
\hline Pneumonia & 13.0 & 16.5 & -0.10 & 16.2 & 15.7 & 0.01 \\
\hline Psoriasis & 1.2 & 1.0 & 0.02 & 1.1 & 1.3 & -0.02 \\
\hline Renal impairment & 18.8 & 27.1 & -0.20 & 25.0 & 24.6 & 0.01 \\
\hline Rheumatoid arthritis & 4.4 & 4.5 & -0.01 & 5.0 & 4.6 & 0.02 \\
\hline Schizophrenia & 0.6 & 0.7 & -0.01 & 0.7 & 0.9 & -0.02 \\
\hline Ulcerative colitis & 0.8 & 1.0 & -0.02 & 0.9 & 0.9 & 0.00 \\
\hline Urinary tract infectious disease & 20.5 & 26.5 & -0.14 & 25.1 & 25.1 & 0.00 \\
\hline Viral hepatitis C & 0.5 & 0.6 & -0.01 & 0.5 & 0.8 & -0.03 \\
\hline Visual system disorder & 28.1 & 29.2 & -0.02 & 29.4 & 29.2 & 0.00 \\
\hline \multicolumn{7}{|l|}{ Medical history: cardiovascular disease } \\
\hline Cerebrovascular disease & 8.6 & 13.2 & -0.15 & 11.1 & 11.2 & 0.00 \\
\hline Coronary arteriosclerosis & 13.1 & 16.4 & -0.09 & 16.0 & 15.7 & 0.01 \\
\hline
\end{tabular}


Table 3 (continued)

\begin{tabular}{|c|c|c|c|c|c|c|}
\hline \multirow[t]{3}{*}{ Characteristic } & \multicolumn{3}{|c|}{ Before matching } & \multicolumn{3}{|c|}{ After matching } \\
\hline & \multirow{2}{*}{$\begin{array}{l}\text { Rivaroxaban } \\
(n=14,480)^{\mathrm{a}} \\
\%\end{array}$} & \multicolumn{2}{|l|}{$\begin{array}{l}\text { Warfarin } \\
(n=25,972)^{\mathrm{b}}\end{array}$} & \multirow{2}{*}{$\begin{array}{l}\text { Rivaroxaban } \\
(n=6921) \\
\%\end{array}$} & \multirow{2}{*}{$\begin{array}{l}\text { Warfarin } \\
(n=6921) \\
\%\end{array}$} & \multirow[b]{2}{*}{ Std. diff } \\
\hline & & $\%$ & Std. diff & & & \\
\hline Heart disease & 44.8 & 52.1 & -0.15 & 51.6 & 51.0 & 0.01 \\
\hline Heart failure & 16.2 & 19.5 & -0.08 & 19.9 & 19.8 & 0.00 \\
\hline Ischemic heart disease & 10.0 & 12.8 & -0.09 & 12.7 & 12.2 & 0.02 \\
\hline Peripheral vascular disease & 10.2 & 12.1 & -0.06 & 11.8 & 11.3 & 0.01 \\
\hline Venous thrombosis & 73.4 & 74.8 & -0.03 & 72.5 & 73.1 & -0.01 \\
\hline \multicolumn{7}{|l|}{ Medical history: neoplasms } \\
\hline Hematologic neoplasm & 6.8 & 5.5 & 0.05 & 6.6 & 6.5 & 0.01 \\
\hline Malignant lymphoma & 1.8 & 1.5 & 0.02 & 1.8 & 1.6 & 0.02 \\
\hline Malignant neoplasm of anorectum & 1.2 & 1.2 & 0.00 & 1.1 & 1.3 & -0.02 \\
\hline Malignant neoplastic disease & 24.9 & 22.5 & 0.06 & 24.5 & 24.5 & 0.00 \\
\hline Malignant tumor of breast & 8.0 & 6.8 & 0.05 & 7.2 & 7.7 & -0.02 \\
\hline Malignant tumor of colon & 2.4 & 2.4 & 0.00 & 2.4 & 2.7 & -0.02 \\
\hline Malignant tumor of lung & 4.8 & 3.6 & 0.06 & 4.7 & 4.2 & 0.02 \\
\hline Malignant tumor of urinary bladder & 0.5 & 0.5 & 0.00 & 0.5 & 0.5 & 0.01 \\
\hline Primary malignant neoplasm of prostate & 0.0 & 0.0 & 0.00 & $<0.1$ & $<0.1$ & 0.01 \\
\hline \multicolumn{7}{|l|}{ Medication use } \\
\hline Agents acting on the renin-angiotensin system & 39.5 & 42.7 & -0.06 & 43.3 & 42.9 & 0.01 \\
\hline Antibacterials for systemic use & 58.7 & 57.9 & 0.02 & 59.4 & 59.2 & 0.00 \\
\hline Antidepressants & 35.6 & 35.9 & 0.00 & 37.5 & 36.9 & 0.01 \\
\hline Antiepileptics & 25.2 & 23.9 & 0.03 & 26.5 & 26.8 & -0.01 \\
\hline Antiinflammatory and antirheumatic products & 27.1 & 24.1 & 0.07 & 25.8 & 25.6 & 0.00 \\
\hline Antineoplastic agents & 12.0 & 9.8 & 0.07 & 11.3 & 10.7 & 0.02 \\
\hline Antipsoriatics & 0.4 & 1.0 & -0.07 & 0.5 & 0.9 & -0.04 \\
\hline Antithrombotic agents & 23.0 & 54.4 & -0.68 & 35.0 & 32.9 & 0.04 \\
\hline Beta-blocking agents & 29.4 & 34.7 & -0.11 & 33.3 & 32.5 & 0.02 \\
\hline Calcium channel blockers & 21.0 & 25.7 & -0.11 & 24.0 & 24.1 & 0.00 \\
\hline Diuretics & 37.3 & 42.6 & -0.11 & 41.4 & 40.7 & 0.02 \\
\hline Drugs for acid-related disorders & 35.2 & 37.6 & -0.05 & 38.5 & 39.1 & -0.01 \\
\hline Drugs for obstructive airway diseases & 27.9 & 27.6 & 0.01 & 28.9 & 28.9 & 0.00 \\
\hline Drugs used in diabetes mellitus & 17.8 & 20.4 & -0.06 & 20.0 & 19.6 & 0.01 \\
\hline Immunosuppressants & 5.3 & 5.1 & 0.01 & 5.5 & 5.6 & 0.00 \\
\hline Lipid-modifying agents & 37.2 & 40.6 & -0.07 & 40.9 & 40.0 & 0.02 \\
\hline Opioids & 40.6 & 40.0 & 0.01 & 42.3 & 42.2 & 0.00 \\
\hline Psycholeptics & 36.8 & 33.9 & 0.06 & 37.6 & 37.3 & 0.01 \\
\hline $\begin{array}{l}\text { Psychostimulants, agents used for attention- } \\
\text { deficit/hyperactivity disorder and nootropics }\end{array}$ & 2.7 & 2.1 & 0.04 & 2.4 & 2.4 & 0.00 \\
\hline Risk scores & Mean & Mean & Std. diff & Mean & Mean & Std. diff \\
\hline Charlson Comorbidity Index & 4.03 & 4.31 & -0.08 & 4.46 & 4.41 & 0.01 \\
\hline $\mathrm{CHA}_{2} \mathrm{DS}_{2}$-VASc & 3.69 & 4.09 & -0.22 & 4.04 & 4.04 & 0.00 \\
\hline
\end{tabular}

$\mathrm{CHA}_{2} \mathrm{DS}_{2}-V A S c$ stroke risk index, Std. diff standardized difference

${ }^{a}$ In the rivaroxaban group, 9 patients were excluded from the study for not having $\geq 1$ day time-at-risk

${ }^{b}$ In the warfarin group, 29 patients were excluded from the study for not having $\geq 1$ day time-at-risk and 4395 patients were excluded when restricting patients to the calendar time when both exposures were observed in the database 


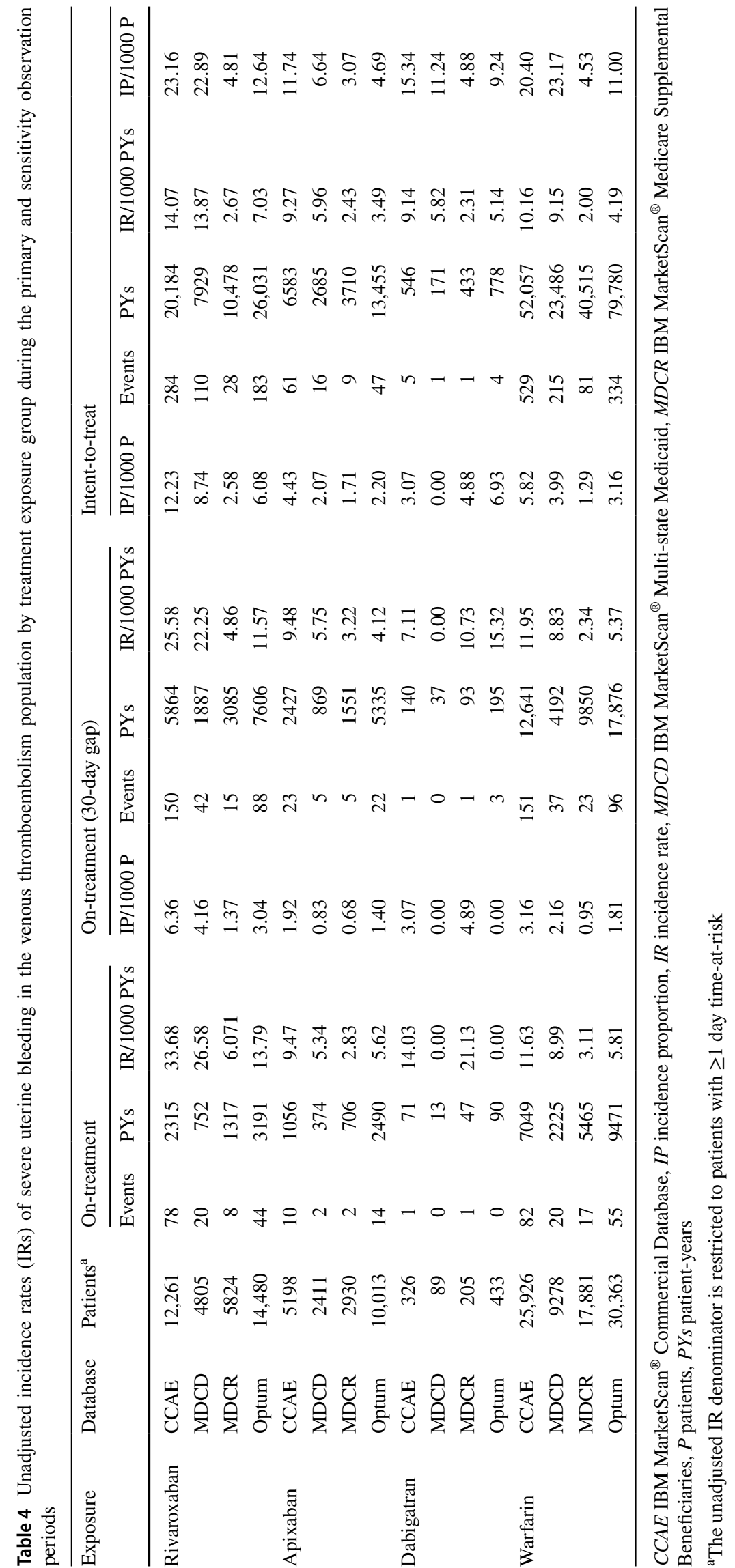


Fig. 3 Calibrated hazard ratios and calibrated $95 \%$ confidence intervals (CIs) for each pairwise comparison during primary and sensitivity observation periods in the venous thromboembolism (VTE) population after 1:1 propensity score matching: (a) rivaroxaban vs warfarin;

(b) apixaban vs warfarin; and

(c) rivaroxaban vs apixaban.

Estimates are reported for pairwise comparisons in databases where study diagnostic passed. Note that limited exposures precluded producing estimates for comparisons including dabigatran in the VTE population. Summary meta-analytic estimates are reported where $I^{2}<40 \%$. CCAE IBM MarketScan ${ }^{\circledR}$ Commercial Database, $c H R$ calibrated hazard ratio, ITT intent-to-treat, $M D C D$ IBM MarketScan ${ }^{\circledR}$

Multi-state Medicaid, $M D C R$

IBM MarketScan ${ }^{\circledR}$ Medicare

Supplemental

Beneficiaries

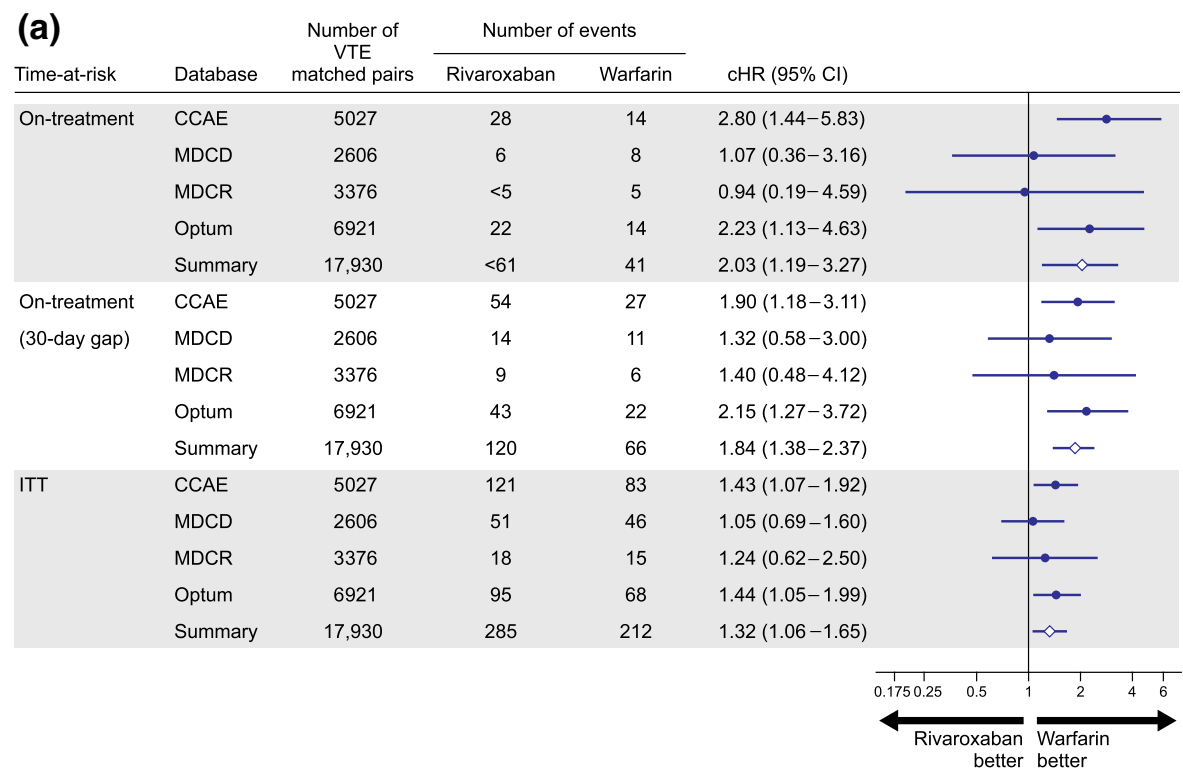

(b)

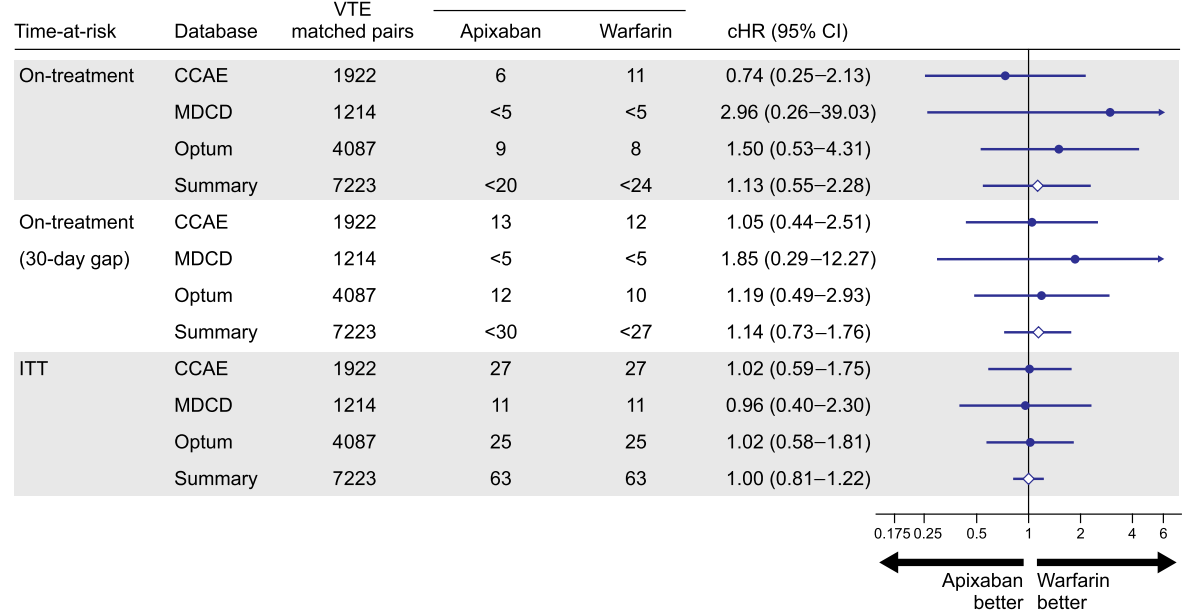

(c)

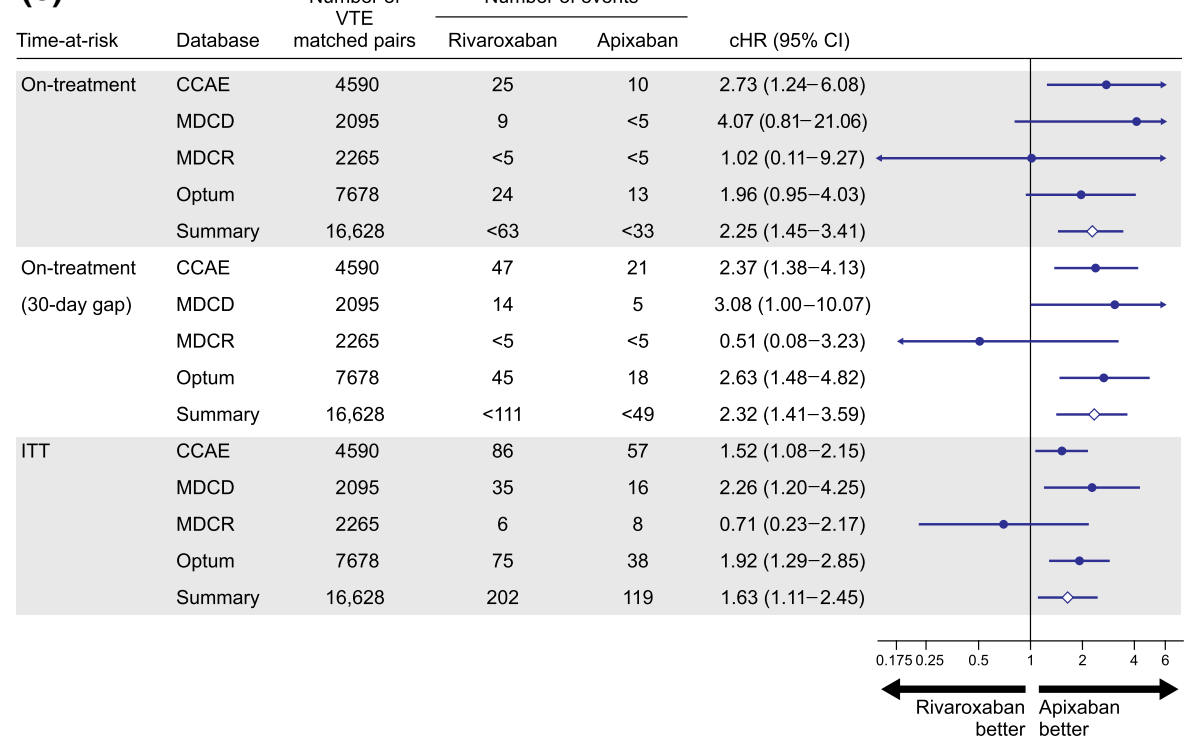


by results obtained from Optum and CCAE data sources. A potential explanation for the different observations may be that the on-treatment analysis is more restrictive, whereas the ITT analysis may include outcome events that are not associated with the treatment, particularly if they occurred long after the treatment discontinuation. In addition, while we observed differences in the statistical significance of the estimates across the different time-at-risk specifications, the estimates were directionally consistent.

Clinical trials of anticoagulant therapies in AF populations report bleeding adverse events, often referred to as major bleeding and clinically relevant non-major bleeding based on the definitions from the International Society on Thrombosis and Haemostasis [12]. Events may include gastrointestinal bleeding, intracranial bleeding, and bleeding from other sites, such as uterine bleeding. Results of these analyses have demonstrated comparable or improved overall major bleeding rates for DOACs vs warfarin $[4,26,27]$. A systematic review and meta-analysis of safety data from controlled trials of patients with atrial fibrillation determined no difference in the major bleeding risk between rivaroxaban or dabigatran compared with vitamin $\mathrm{K}$ antagonists but a $31 \%$ risk reduction with apixaban vs vitamin $\mathrm{K}$ antagonists based on the ARISTOLE trial [28].

Several observational studies have compared bleeding rates, but not specifically uterine bleeding, among DOACs and vitamin $\mathrm{K}$ antagonists in the AF population with varying results. No significant differences in short-term bleeding were identified between new users of dabigatran and a vitamin K antagonist (HR 0.88; 95\% CI 0.64-1.21) and between new users of rivaroxaban and a vitamin $\mathrm{K}$ antagonist (HR 0.98; 95\% CI 0.64-1.51) [24] or among patients with AF who switched from vitamin $\mathrm{K}$ antagonists to dabigatran or rivaroxaban [99 (1\%)] vs those who did not switch therapy [193 (2\%); $p=0.54$ ] [29]. A significant difference in major bleeding risk among matched cohorts of patients with $\mathrm{AF}$ was identified between rivaroxaban and apixaban $(4.2 \%$ vs 2.4\%; HR 1.82; 95\% CI 1.36-2.43) but not between new users of rivaroxaban and warfarin (4.9\% vs $5.1 \%$; HR 0.98 ; 95\% CI 0.83-1.17) or between new users of rivaroxaban and dabigatran (3.3\% vs 3.1\%; HR 1.05; 95\% CI 0.74-1.49) [30]. Optum data reported an increased risk of major bleeding among users of rivaroxaban compared to both apixaban (HR 2.56; 95\% CI 1.85-3.57; $p<0.001$ [data were inverted to provide the comparison of rivaroxaban vs apixaban]) and dabigatran (HR 1.30; 95\% CI 1.10-1.53; $p<0.01$ ) [31]. A similar PS 1:1 matching analysis using Medicare Advantage coverage found that apixaban was associated with a significantly lower risk of major bleeding than rivaroxaban and warfarin (HR 0.49; 95\% CI 0.41-0.59; $p<0.001$ and HR $0.53 ; 95 \%$ CI $0.45-0.63 ; p<0.001$, respectively) [32]. These data suggest possible differences in bleeding risk associated with anticoagulants in patients with AF.
Our results show that the risk of severe uterine bleeding was higher among patients with VTE than in patients with AF across all treatment groups. Further, rivaroxaban was associated with a statistically significant increased risk of severe uterine bleeding compared to both warfarin and apixaban in patients with VTE. These results were consistent across sensitivity analyses both over longer time-atrisk periods and using different PS matching approaches. A trend toward an increased risk of severe uterine bleeding was observed for new users of apixaban compared to warfarin during the primary observation period, but estimates attenuated with a longer time-at-risk duration. The higher risk of severe uterine bleeding in general, and the more evident relative risk difference for rivaroxaban users in the VTE population, is likely because of the age of the population. Female patients with VTE are more likely to be of reproductive age compared to female patients with AF, putting them at higher risk for severe uterine bleeding. Our findings are consistent with the results of prior studies. In an analysis of the EINSTEIN-DVT and EINSTEIN-PE clinical trials, women $<60$ years of age had more frequent abnormal uterine bleeding when treated with rivaroxaban compared with enoxaparin/vitamin K antagonist (HR 2.13; 95\% CI 1.57-2.89) [7]. The AMPLIFY trial reported that clinically relevant non-major vaginal bleeding occurred in similar proportions of women receiving apixaban and those receiving enoxaparin/warfarin (2.5\% vs $2.1 \%$, respectively; odds ratio $1.2,95 \%$ CI 0.7-2.0) [8]. Moreover, in a single-center retrospective study, the risk of abnormal uterine bleeding was assessed among 104 female patients with VTE of reproductive age who were treated with either rivaroxaban or a vitamin $\mathrm{K}$ antagonist [33]. Patients receiving rivaroxaban were more likely to report an unscheduled contact with a physician (41\% vs $25 \% ; p=0.096)$ and an increased need for menorrhagia-related medical or surgical intervention (25\% vs $7.7 \% ; p=0.032$ ) compared with women receiving a vitamin $\mathrm{K}$ antagonist [34].

This study provides real-world evidence on the comparative risk of severe uterine bleeding among anticoagulation treatments and across different patient populations. Past studies have suggested that the risk of bleeding in general and, specifically uterine bleeding, may vary for different indications of anticoagulation. However, existing comparative safety studies tended to focus on evaluating an outcome of general severe bleeding in the AF population and an outcome of abnormal uterine bleeding for the VTE population. Our results provide important evidence on considering patient characteristics and the indication of anticoagulation when balancing the risk and benefits of alternative anticoagulation treatments. Patients with AF are mostly aged older than 60 years and therefore beyond the reproductive age, but many female patients receiving DOACs for VTE are younger and can potentially be affected by this complication. Women 
requiring anticoagulation should be counseled and informed to assist in making appropriate treatment choices. The personalized management strategy for patients who might be at a higher risk of bleeding, such as those with heavy menstrual bleeding, requires careful evaluation of the risk vs the benefit of continued anticoagulation considering the severity of bleeding as well as individual thrombotic risk.

This study evaluated outcomes from large observational datasets that include a wide variety of clinical care settings of insured US adults and provide complementary information about anticoagulation treatment utilization and effects in routine clinical practice. The outcome under study, severe uterine bleed, is very rare and it is unlikely that randomized and/or prospective studies would have enough events and statistical power for a meaningful risk evaluation. In addition, the International Society on Thrombosis and Haemostasis definition of clinically relevant non-major bleeding does not include heavy menstrual bleeding, a less severe but more common bleeding outcome, which also contributes to the lack of uterine bleeding data and related research. Finally, our study allowed for direct estimation of incidence rates following the exposure to four investigated drugs, and the new-user cohort design captured early events following treatment exposures while avoiding confounding from previous treatment effects. This design also allowed for a clear exposure index date designation.

The following limitations of this study need to be considered. First, indication and outcome misclassification are a concern in studies using administrative databases because diagnosis codes intended for reimbursement cannot verify patient clinical condition with certainty. In addition, to our knowledge, the diagnostic and procedure codes used to define the outcome were not previously validated, which could be subject to measurement and classification errors. Random misclassification can attenuate the results toward the null. Second, as with any observational study, residual confounding may still be present. Some confounders may be unmeasured or inadequately represented in claims data, including weight, smoking status, many clinical measurements, and lifestyle behaviors, such as diet and exercise. Adjustment by PS cannot completely remove bias because of unmeasured or incorrectly specified confounders. It is worth mentioning that upon investigating the baseline characteristics of the treatment group (ESM Online Resources 3, 5, and 7), new users of apixaban tended to have higher comorbidity compared to the other three treatment groups. However, our diagnostics for all the presented results show that we achieved acceptable balance after PS matching (https://data.ohdsi.org/Doacs WarfarinSub/; "Covariate Balance" tab). In addition, our results were empirically calibrated on the basis of negative and positive controls and indicated sufficient control over residual error and unmeasured confounding inherent to the study design and data. It was noted that clinical equipoise [35] for some of the treatment comparisons was far below $50 \%$ based on the PS distribution, for example, rivaroxaban vs warfarin in the VTE population. When clinical equipoise is above $50 \%$, the estimate from a treatment comparison may represent evidence that is reasonably generalizable to most patients treated in clinical practice. In contrast, when clinical equipoise is below 50\%, the estimates may be less generalizable to the population treated in clinical practice because many patients in the comparison are incomparable and discarded from analysis. However, reduced generalizability does not undermine the strong internal validity of a finding where a subset of patients is well matched on the PS. Clinical equipoise varied considerably in this study because we made multiple exposure comparisons across multiple databases; therefore, interpretation of the results requires careful assessment of both generalizability and internal validity. Finally, some treatment comparisons exhibited bimodal and multimodal PS distributions, for example, rivaroxaban vs apixaban in the AF population. In these instances, the modal peaks represent a strong predictive contribution of one or a few covariates in the PS model.

\section{Conclusions}

The incidence of severe uterine bleeding was low in the AF and VTE populations studied. Among patients with AF receiving anticoagulants, the risk of severe uterine bleeding was moderately increased for rivaroxaban new users compared with new users of warfarin, apixaban, and dabigatran. This risk was sensitive to the time-at-risk period. In the younger VTE population, a consistent increased risk of severe uterine bleeding was observed for rivaroxaban in comparison to apixaban and warfarin. There was no strong evidence suggesting differential severe uterine bleeding risk between apixaban, dabigatran, and warfarin in either population. Personalized management strategies with careful evaluation of benefits and risks are required for women who need antithrombotic therapy.

Supplementary Information The online version contains supplementary material available at https://doi.org/10.1007/s40264-021-01060-4.

Acknowledgements Medical writing support was provided by Michelle McDermott, PharmD, of MedErgy, and was funded by Janssen Scientific Affairs, LLC.

\section{Declarations}

Funding This study was funded by Janssen Research \& Development, LLC. 
Conflict of interest All authors are full-time employees of Janssen Research \& Development, LLC and may be stockholders of Johnson \& Johnson.

Ethics approval Analyses of de-identified, publicly available data do not constitute human subjects research and, as such, do not require institutional review board review/approval.

Consent to participate Not applicable.

Consent for publication Not applicable.

Availability of data and material The full result set with associated study diagnostics is publicly available in an interactive web application at https://data.ohdsi.org/DoacsWarfarinSub/.

Code availability The code is available at https://github.com/ohdsi -studies/DoacsWarfarinSub/tree/master/DoacsWarfarinSub.

Authors' contributions JW, AS, HQT, LL, SW, PW, GR, AF, LW, ZY, and $\mathrm{EB}$ contributed to the conception of the study, the design, interpretation of the results, writing and editing of the manuscript, and final approval of the manuscript. JW and AS conducted the data analysis.

Open Access This article is licensed under a Creative Commons Attribution-NonCommercial 4.0 International License, which permits any non-commercial use, sharing, adaptation, distribution and reproduction in any medium or format, as long as you give appropriate credit to the original author(s) and the source, provide a link to the Creative Commons licence, and indicate if changes were made. The images or other third party material in this article are included in the article's Creative Commons licence, unless indicated otherwise in a credit line to the material. If material is not included in the article's Creative Commons licence and your intended use is not permitted by statutory regulation or exceeds the permitted use, you will need to obtain permission directly from the copyright holder. To view a copy of this licence, visit http://creativecommons.org/licenses/by-nc/4.0/.

\section{References}

1. Xarelto ${ }^{\circledR}$ (rivaroxaban) tablets, for oral use [package insert]. Titusville: Janssen Pharmaceuticals, Inc., 2019.

2. Eliquis ${ }^{\circledR}$ (apixaban) tablets for oral use [package insert]. Princeton: Bristol Myers Squibb Company, 2012.

3. Pradaxa ${ }^{\circledR}$ (dabigatran etexilate mesylate) capsules, for oral use [package insert]. Ridgefield: Boehringer Ingelheim Pharmaceuticals, Inc., 2015.

4. Patel MR, Mahaffey KW, Garg J, Pan G, Singer DE, Hacke W, et al. Rivaroxaban versus warfarin in nonvalvular atrial fibrillation. N Engl J Med. 2011;365(10):883-91.

5. Sherwood MW, Nessel CC, Hellkamp AS, Mahaffey KW, Piccini JP, Suh EY, et al. Gastrointestinal bleeding in patients with atrial fibrillation treated with rivaroxaban or warfarin: ROCKET AF trial. J Am Coll Cardiol. 2015;66(21):2271-81.

6. Bistervels IM, Scheres LJJ, Hamulyak EN, Middeldorp S. Sex matters: practice 5P's when treating young women with venous thromboembolism. J Thromb Haemost. 2019;17(9):1417-29.

7. Martinelli I, Lensing AW, Middeldorp S, Levi M, Beyer-Westendorf J, van Bellen B, et al. Recurrent venous thromboembolism and abnormal uterine bleeding with anticoagulant and hormone therapy use. Blood. 2016;127(11):1417-25.
8. Brekelmans MP, Scheres LJ, Bleker SM, Hutten BA, Timmermans A, Buller HR, et al. Abnormal vaginal bleeding in women with venous thromboembolism treated with apixaban or warfarin. Thromb Haemost. 2017;117(4):809-15.

9. Huisman MV, Ferreira M, Feuring M, Fraessdorf M, Klok FA. Less abnormal uterine bleeding with dabigatran than warfarin in women treated for acute venous thromboembolism. J Thromb Haemost. 2018;16(9):1775-8.

10. Scheres LJJ, Brekelmans MPA, Ageno W, Ay C, Buller HR, Eichinger $\mathrm{S}$, et al. Abnormal vaginal bleeding in women of reproductive age treated with edoxaban or warfarin for venous thromboembolism: a post hoc analysis of the Hokusai-VTE study. BJOG. 2018;125:1581-9.

11. Munro MG, Critchley HO, Fraser IS, Figo Menstrual Disorders Committee. The two FIGO systems for normal and abnormal uterine bleeding symptoms and classification of causes of abnormal uterine bleeding in the reproductive years: 2018 revisions. Int J Gynaecol Obstet. 2018;143(3):393-408.

12. Kaatz S, Ahmad D, Spyropoulos AC, Schulman S, Subcommittee on Control of Anticoagulation. Definition of clinically relevant non-major bleeding in studies of anticoagulants in atrial fibrillation and venous thromboembolic disease in non-surgical patients: communication from the SSC of the ISTH. J Thromb Haemost. 2015;13(11):2119-26.

13. Voss EA, Makadia R, Matcho A, Ma Q, Knoll C, Schuemie M, et al. Feasibility and utility of applications of the common data model to multiple, disparate observational health databases. J Am Med Inform Assoc. 2015;22(3):553-64.

14. Tian Y, Schuemie MJ, Suchard MA. Evaluating large-scale propensity score performance through real-world and synthetic data experiments. Int J Epidemiol. 2018;47(6):2005-14.

15. Tibshirani R. Regression shrinkage and selection via the lasso. J R Stat Soc Series B Stat Methodol. 1996;58(1):267-328.

16. Rassen JA, Shelat AA, Myers J, Glynn RJ, Rothman KJ, Schneeweiss S. One-to-many propensity score matching in cohort studies. Pharmacoepidemiol Drug Saf. 2012;21(Suppl. 2):69-80.

17. Austin PC. Balance diagnostics for comparing the distribution of baseline covariates between treatment groups in propensityscore matched samples. Stat Med. 2009;28(25):3083-107.

18. Schuemie MJ, Cepeda MS, Suchard MA, Yang J, Tian Y, Schuler A, et al. How confident are we about observational findings in health care: a benchmark study. Harv Data Sci Rev. 2020. https://doi.org/10.1162/99608f92.147cc28e.

19. Wang SV, He M, Jin Y, Wyss R, Shin H, Ma Y, et al. A review of the performance of different methods for propensity score matched subgroup analyses and a summary of their application in peer-reviewed research studies. Pharmacoepidemiol Drug Saf. 2017;26(12):1507-12.

20. Schuemie MJ, Ryan PB, DuMouchel W, Suchard MA, Madigan D. Interpreting observational studies: why empirical calibration is needed to correct $p$ values. Stat Med. 2014;33(2):209-18.

21. Schuemie MJ, Hripcsak G, Ryan PB, Madigan D, Suchard MA. Empirical confidence interval calibration for population-level effect estimation studies in observational healthcare data. Proc Natl Acad Sci USA. 2018;115(11):2571-7.

22. Higgins JP, Thompson SG, Deeks JJ, Altman DG. Measuring inconsistency in meta-analyses. BMJ. 2003;327(7414):557-60.

23. Piccini JP, Stevens SR, Lokhnygina Y, Patel MR, Halperin JL, Singer DE, et al. Outcomes after cardioversion and atrial fibrillation ablation in patients treated with rivaroxaban and warfarin in the ROCKET AF trial. J Am Coll Cardiol. 2013;61(19):1998-2006.

24. Maura G, Blotiere PO, Bouillon K, Billionnet C, Ricordeau P, Alla F, et al. Comparison of the short-term risk of bleeding and arterial thromboembolic events in nonvalvular atrial fibrillation patients newly treated with dabigatran or rivaroxaban versus 
vitamin K antagonists: a French nationwide propensity-matched cohort study. Circulation. 2015;132(13):1252-60.

25. Suchard MA, Simpson SE, Zorych I, Ryan P, Madigan D. Massive parallelization of serial inference algorithms for a complex generalized linear model. ACM Trans Model Comput Simul. 2013. https://doi.org/10.1145/2414416.2414791.

26. Hylek EM, Held C, Alexander JH, Lopes LR, De Caterina R, Wojdyla DM, et al. Major bleeding in patients with atrial fibrillation receiving apixaban or warfarin: the ARISTOTLE trial (Apixaban for Reduction in Stroke and Other Thromboembolic Events in Atrial Fibrillation): predictors, characteristics, and clinical outcomes. J Am Coll Cardiol. 2014;63(20):2141-7.

27. Connolly SJ, Ezekowitz MD, Yusuf S, Eikelboom J, Oldgren J, Parekh A, et al. Dabigatran versus warfarin in patients with atrial fibrillation. N Engl J Med. 2009;361(12):1139-51.

28. Almutairi AR, Zhou L, Gellad WF, Lee JK, Slack MK, Martin $\mathrm{JR}$, et al. Effectiveness and safety of non-vitamin $\mathrm{K}$ antagonist oral anticoagulants for atrial fibrillation and venous thromboembolism: a systematic review and meta-analyses. Clin Ther. 2017;39(7):1456-78.e36.

29. Bouillon K, Bertrand M, Maura G, Blotière P-O, Ricordeau P, Zureik M. Risk of bleeding and arterial thromboembolism in patients with non-valvular atrial fibrillation either maintained on a vitamin $\mathrm{K}$ antagonist or switched to a non-vitamin $\mathrm{K}$-antagonist oral anticoagulant: a retrospective, matched-cohort study. Lancet Haematol. 2015;2(4):e150-9.

30. Lip GY, Keshishian A, Kamble S, Pan X, Mardekian J, Horblyuk $\mathrm{R}$, et al. Real-world comparison of major bleeding risk among non-valvular atrial fibrillation patients initiated on apixaban, dabigatran, rivaroxaban, or warfarin: a propensity score matched analysis. Thromb Haemost. 2016;116(5):975-86.

31. Noseworthy PA, Yao X, Abraham NS, Sangaralingham LR, McBane RD, Shah ND. Direct comparison of dabigatran, rivaroxaban, and apixaban for effectiveness and safety in nonvalvular atrial fibrillation. Chest. 2016;150(6):1302-12.

32. Deitelzweig S, Luo X, Gupta K, Trocio J, Mardekian J, Curtice $\mathrm{T}$, et al. Comparison of effectiveness and safety of treatment with apixaban vs. other oral anticoagulants among elderly nonvalvular atrial fibrillation patients. Curr Med Res Opin. 2017;33(10):1745-54.

33. Kohsaka S, Katada J, Saito K, Jenkins A, Li B, Mardekian J, et al. Safety and effectiveness of non-vitamin K oral anticoagulants versus warfarin in real-world patients with non-valvular atrial fibrillation: a retrospective analysis of contemporary Japanese administrative claims data. Open Heart. 2020;7(1):e001232.

34. De Crem N, Peerlinck K, Vanassche T, Vanheule K, Debaveye B, Middeldorp S, et al. Abnormal uterine bleeding in VTE patients treated with rivaroxaban compared to vitamin $\mathrm{K}$ antagonists. Thromb Res. 2015;136(4):749-53.

35. Walker A, Patrick A, Lauer M, Hornbrook M, Marin M, Platt R, Roger V, Stang P, Schneeweiss S. A tool for assessing the feasibility of comparative effectiveness research. Comp Eff Res. 2013;3:11-20. 